\title{
Vom Platz der Siege ins Künstlerhaus
}

Dass aller Fortschritt in der Informationstechnologie, die zunehmende und bald vielleicht grenzenlose Verfügbarkeit wissenschaftlicher Literatur über das Netz, nicht zur heimischen Vereinzelung der Forscher und zum massiven Publikumsschwund in den öffentlichen Bibliotheken geführt hat, sondern, im Gegenteil, allerorten neue Bibliotheken entstehen und deren Lesesäle eine ungeahnte Revitalisierung erleben, ist der schönste Beweis für die Unverzichtbarkeit von wissenschaftlichen Interaktionsräumen, in denen man ebenso leicht ins Buch wie auch wieder heraus finden und ins Gespräch treten kann. Und weil kaum jemand vom Sinn und Nutzen öffentlichen wissenschaftlichen Raums überzeugter ist und sich immer wieder erfolgreich um ihn bemüht hat, als Horst Bredekamp, sei, auch weil er darin anderen zum Vorbild geworden ist, der im Herbst 2011 erfolgte Umzug des Deutschen Forums für Kunstgeschichte in Paris hier exemplarisch aufgeführt.

Das Forum hat über viele Jahre hinweg an der Pariser Place des Victoires eben diese Verbindung aus Studiolo und Parlour zu verwirklichen versucht, um hier die sprachlich und kulturell je unterschiedlich geprägte Kunstgeschichte des französischen und des deutschsprachigen Raums in ein kritisch-produktives Verhältnis zueinander zu setzen. Längst schon aber beschränkt sich dieser Dialog nicht auf binationale Dimensionen allein. Dass das Deutsche Forum inzwischen als lebhafter carrefour einer zumal in Paris sich zunehmend formierenden, international operierenden Kunstgeschichte wahrgenommen wird, die sich darüber hinaus auch bewusst anderen Fachorientierungen öffnet, ist nicht zuletzt der polyglotten Atmosphäre geschuldet, die sich in seinen immer wieder zu einem regelrechten wissenschaftlichen Salon verdichtenden Räumen entfaltet hat.

Hinter den majestätischen, von Jules Hardouin-Mansart entworfenen Fassaden mit Kolossalordnung und Blick in das Platzrund, hat das Deutsche Forum für Kunstgeschichte dieser akademischen Gesprächskultur von Anfang an die größte Bedeutung zugemessen. Die Würdeformeln des Platzes, seiner Dekoration und Architektur, stehen einmal für die wechselvolle Geschichte der Anlage, die in nuce eine Stadt- und 
Bildgeschichte von Paris seit dem Grand siècle abbildet. Auf Initiative des Marschalls de la Feuillade wurde sie zu Ehren Ludwigs XIV. Ende der 1680er Jahre geschaffen. Im Zuge der Revolution geriet die sie beherrschende Königs-Statue zu einem kapitalen Beispiel ikonoklastischer Energie, um dann von einer Statue des Generals Desaix ersetzt zu werden, welche ihrerseits zur Zeit der Restauration einem Reiterdenkmal Ludwigs XIV. von François-Joseph Bosio (1822) weichen musste. Zugleich ist dieses in solch anschaulicher Geschlossenheit singuläre Ambiente aber auch zur inspirierenden Rahmung eines vielstimmigen Gesprächs geworden, das sich sowohl diesen Bild- und Raumfindungen unmittelbar zugewandt hat, als auch, von hier aus, weit und weiter ausgegriffen hat.

Wenn unter den zahllosen Veranstaltungen, die in den Räumen des Deutschen Forums für Kunstgeschichte an der Place des Victoires seither stattgefunden haben, hier jener Studientag besonders hervorgehoben werden soll, den die Kolleg-Forschergruppe Bildakt und Verkörperung gemeinsam mit den Stipendiaten des der „Poiesis“ gewidmeten Jahresthemas des Forums im Herbst 2009 ausgerichtet hat, dann, weil dieser einen einschlägigeren Ort wohl nicht hätte finden können. Die Macht und Kraft der Bildwerke, ihre durch und durch poietische Wirkkraft bindet sich ja exemplarisch an diesen Platz und die dynamische Entfaltung der damals in besonderer Intensität geführten Diskussionen hat den genius loci ein weiteres Mal bekräftigt. Der von Horst Bredekamp und dem unvergessenen John Michael Krois seinerzeit gemeinsam bestrittene öffentliche Abendvortrag schließlich ist allen Teilnehmern als Beispiel einer gelungenen wissenschaftlichen Synchronie, wie sie das Forum herzustellen sich immer wieder bemüht, in schönster Erinnerung.

Für die Vitalität und Attraktivität des Forums spricht, dass seine angestammte Lokalität bald schon nicht mehr ausgereicht hat und die zum Schluss sich über drei Gebäude auf fünf Etagen verteilenden Räume, inklusive ausgelagertem Buchmagazin, den Anforderungen an einen wirkungsvollen Bibliotheks- und Institutsbetrieb sowie der gestiegenen Mitarbeiterzahl nicht mehr annähernd genügt haben. Ganz bewusst soll betont werden, dass es das Bundesministerium für Bildung und Forschung auszeichnet, dass es von der Notwendigkeit einer adäquaten Domizilierung seiner in der Max Weber-Stiftung-Deutsche Geisteswissenschaftliche Institute im Ausland versammelten Forschungseinrichtungen 
überzeugt ist - der freie Verkehr der Gedanken nämlich benötigt feste Hüllen ebenso sehr, wie immer geschwindere Datenautobahnen. Der dem Deutschen Forum für Kunstgeschichte zum Herbst 2011 zugebilligte Umzug in ein neues Gebäude, das Hôtel Lully, hat die Fläche mehr als verdoppeln lassen. Erstmals ist so die Einrichtung eines eigenen Lesesaals für die in wachsender Zahl auf die Spezialsammlungen des Forums zugreifenden Kollegen und Kolleginnen vor Ort gewährleistet worden, konnten die Büros der Mitarbeiter von Bibliotheksbeständen entlastet und adäquate Arbeitsräume für die Mitarbeiter von Wissenschaft und Verwaltung ebenso bereitgestellt werden, wie für Forscher eingeworbener Drittmittelprojekte oder wissenschaftliche Gäste sowie nicht zuletzt für die Stipendiaten des Forums, für welche die oberste Etage des neuen Baus, das Atelier, reserviert bleibt. In diesem trägt, bei hellem Oberlicht, ein großzügiger Saal zu individueller Konzentration ebenso bei, wie zu integrativer Kommunikation. Im Souterrain verfügt das Forum nun erstmals über einen eigenen Vortragssaal, der mit einer Kapazität von nahezu 70 Plätzen den regelmäßig sehr gut bis stark besuchten Veranstaltungen bereits in den ersten Monaten gerecht geworden ist. Benannt ist er nach Julius Meier-Graefe (1867-1935), womit das Forum einen der zentralen Vermittler im Kunstgeschehen zwischen Frankreich und Deutschland in einer seiner entscheidenden Phasen würdigt.

Bei der Suche nach einem neuen, anzumietenden Domizil wurde, neben der räumlichen Ausdehnung, vor allem auch auf die Lage geachtet. War schon die Unterbringung an der Place des Victoires der Nähe zum Louvre, zum benachbarten Institut national d'histoire de l'art sowie zur alten Bibliothèque Nationale geschuldet, die in Kürze als zentrale französische Forschungsbibliothek zur Kunstgeschichte wiedereröffnen wird, ist auch die nur wenige Gehminuten von der Place des Victoires entfernt liegende No. 45, rue des Petits Champs ideal in einem sich auch international verdichtenden kunsthistorischen Koordinatensystem platziert; erst jüngst hat die US-amerikanische Terra Foundation for American Art Europe dort in unmittelbarer Nähe ihr europäisches Hauptquartier aufgeschlagen.

Das Hôtel Lully verdankt seine Errichtung dem aus Italien stammenden Hofkomponisten Ludwigs XIV., Jean-Baptiste Lully, der für den Erwerb des Terrains und die Errichtung des Baus, so will es die lokale Legende, von Molière mit 11.000 livres unterstützt wurde. Was umso 
pikanter ist, als sich just während der Errichtungszeit (ab 1670) Lully und Molière, die lange Zeit in der neuen Gattung des „Comédie-ballet“ zusammenwirkten, bekanntermaßen endgültig zerstritten. Beauftragter Architekt war Daniel Gittard, ein Schüler von Louis Le Vau, Protégé des Oberaufsehers über die königlichen Finanzen, Nicolas Fouquet, und architecte du roi. Gittard zeichnet für zahlreiche hôtels particuliers in Paris verantwortlich, war an den Grundlegungen für Vaux-le-Vicomte ebenso beteiligt wie an dem Grand Degré in Chantilly oder an den Umbauten der Kirche von Saint-Sulpice in Paris und wurde 1671 eines der ersten Mitglieder der von Ludwig XIV. neu eingerichteten Académie royale d'architecture.

Das Hôtel Lully, das bis 1807 in Besitz der Nachfahren des Komponisten blieb, zeigt den Baustil des Grand siècle heute nur noch an der Fassade. Eine massive Entkernung in den neunziger Jahren des vergangenen Jahrhunderts hat, mit Ausnahme eines kleinen Deckenfreskos, nichts mehr von der einstmals prächtigen Innenausstattung übrig gelassen. Dafür freilich bot sich die Gelegenheit, auf den über einen L-förmig sich erstreckenden Grundrissen sämtlicher Etagen ganz nach Bedarf Raumeinheiten zu schaffen, die den spezifischen Nutzungsanforderungen gehorchen. Eine spektakuläre hölzerne Wendeltreppe verbindet die Stockwerke und ist rasch zum Dreh- und Angelpunkt des Institutsalltags geworden.

Die entlang der rue des Petits Champs und der rue Sainte-Anne sich entlang ziehende Fassade des Hôtel Lully, die zwei ursprünglich separate Bauteile nach außen harmonisierte, zeigt ein rustiziertes Sockelgeschoß, das von Arkaden skandiert wird, deren Schlusssteine skulptierte Masken schmücken. Die von einer kompositen Kolossalordnung zusammengefassten Hauptgeschosse werden von einem durchfensterten Mansardendach bekrönt. Gittard experimentierte an diesem Stadthaus des Musterbeispiels eines Hofkünstlers mit nobilitierenden Bauformen, die den jüngeren und bald mit ihm konkurrierenden Architekten Jules Hardouin-Mansart nachweislich und nachhaltig inspiriert haben. Wenig später sollte dieser sie virtuos weiter entwickeln und namentlich seine Bauten an der königlichen Place des Victoires damit instrumentieren. Es ist, als sei das Forum gleichsam an die Ursprünge der Baukultur der Place des Victoires zurückgezogen - womit sein neues Domizil wie zum Sinnbild von Kontinuität und Wandel geraten ist. 
Kontinuität und Wandel sind es auch, welche die inhaltliche Arbeit des Deutschen Forums für Kunstgeschichte prägen. In den vergangenen Jahren hat die Forschungsarbeit des Forums die traditionell mit dem Haus verbundene Konzentration auf die deutsch-französischen Kunstbeziehungen in Praxis und Kritik in Publikationen und in der Ansiedlung neuer Forschungsprojekte vertieft und verstärkt. Darüber hinaus aber wurde die wechselseitige Kenntnisnahme und Erforschung kunsttheoretischer und methodischer Positionen vorangetrieben, was die deutsch-französische Kunstgeschichte auf gegenstandsbezogener wie diskursiver Ebene neue Perspektiven hat entwickeln lassen. Die erfreuliche Zunahme von französischen Forschungsarbeiten zur Kunst und Kunsttheorie im deutschsprachigen Raum, die zu befördern das Forum sich nicht weniger in der Pflicht sieht, als jene zur französischen Kunst aus den deutschsprachigen Ländern, ist Frucht einer intensiv verfolgten Vermittlungsaktivität, die nicht zuletzt auch in den neuen Bibliotheksräumen in der rue des Petits Champs zuversichtlich gesteigert werden wird. Beide kunsthistorischen Sprach- und Denkkulturen, auch als Alternative zur anglophonen Standardisierung der Wissenschaftssprache, zusammenzubringen, geschieht in der Überzeugung, dass von der Kunst und ihrer Geschichte als wirklichem Ferment einer polyglotten Wissenschaftskultur ausgegangen werden darf.

Am 4. April 2012 ist das Hôtel Lully festlich eingeweiht worden. Es war unser aller Wunsch, dass, neben Pierre Rosenberg, dem ehemaligen Direktor des Louvre und seit Beginn kritisch-solidarischen Freundes des Hauses, Horst Bredekamp zu den versammelten Kollegen und Freunden sprechen solle. Er hat diesem Wunsch bereitwillig entsprochen und in einer dichten Rede auf die kunsthistorischen Verbindungslinien verwiesen, die Deutschland und Frankreich, in Zuneigung und Abgrenzung, aneinanderbinden. Er sprach dabei von Wilhelm von Humboldt und dessen am Pariser Beispiel gereiften Überzeugung eines von der Obrigkeit autonomen Kunstkörpers; von der ebenso lang zurückreichenden wie zeitweise fatalen Auseinandersetzung um die Kunst des Mittelalters und noch vom jungen Marcel Duchamp, dessen Hinwendung zur Welt der Maschinen und Artefakte sich einem 1912 inkognito absolvierten, jetzt erstmals einlässlich rekonstruierten Aufenthalt in München, und namentlich dem Besuch des dortigen Deutschen Museums verdankt. Vor allem auch hat er dem Deutschen Forum für Kunstgeschichte Mut 
gemacht, weil er darauf verwies, dass es, von Thomas Gaehtgens gegründet, aus einem persönlichen, vollständig unabhängigen Forscher- und Vermittlereros entstanden ist. Und dass es dieses Klima nur dann bewahre, wenn es nicht nach seiner Verfasstheit, sondern seinem intellektuellen Anspruch nach eine Institution zu sein beanspruche. Dazu gehört auch das Bewusstsein von Fremdheit und Anziehung.

Dass das Deutsche Forum für Kunstgeschichte Paris nunmehr in einem Haus beherbergt ist, das ein aus der Fremde nach Frankreich gelangter und hier höchste Wirkung entfaltet habender Künstler hat errichten lassen, ist Vermächtnis und Verpflichtung zugleich. Im kleinen, begrünten Innenhof des neuen Gebäudes empfangen, beim ersten Betreten, eine schon zuvor dort gepflanzte Hortensie und ein GinkgoBaum. Das kann unmittelbar die Lyrik Rilkes und Goethes wachrufen, zweier Dichter, die zu Frankreich und namentlich zu Paris in höchster Spannung und schönster Empfänglichkeit standen. Dies soll als ein Zeichen gewertet werden. 


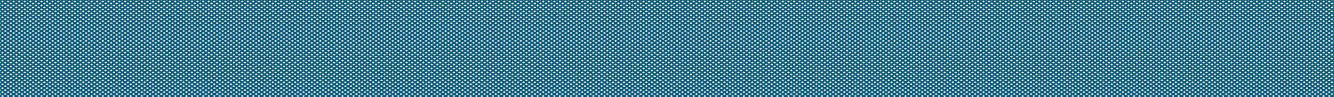

1
1

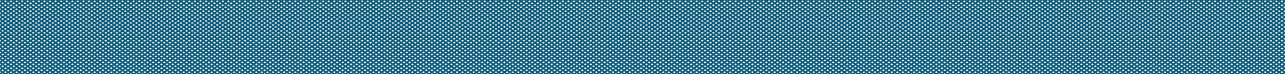
1
1 1
1 1
1

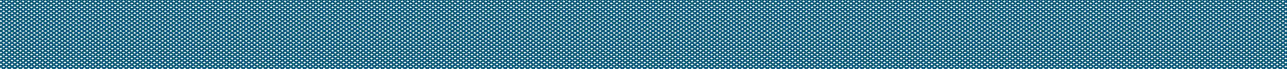

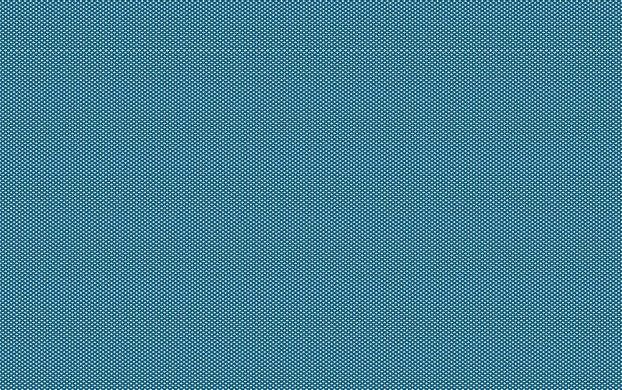

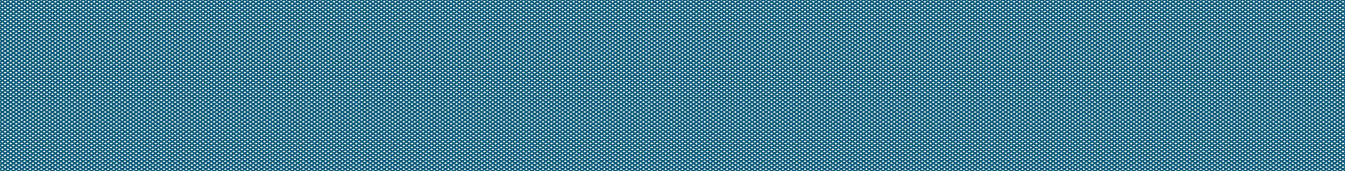
1
1

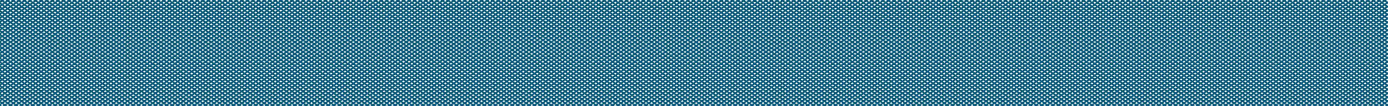

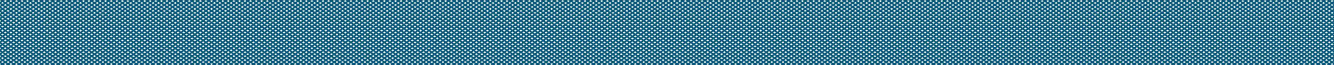
1
1

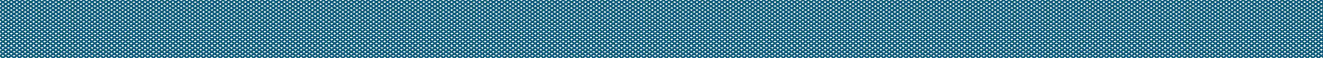

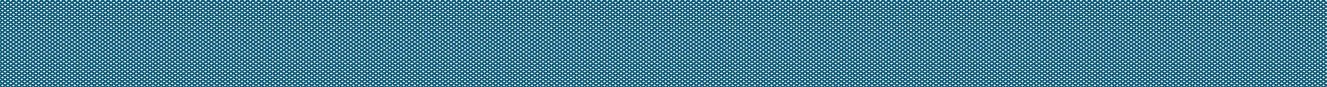
-1
-1 


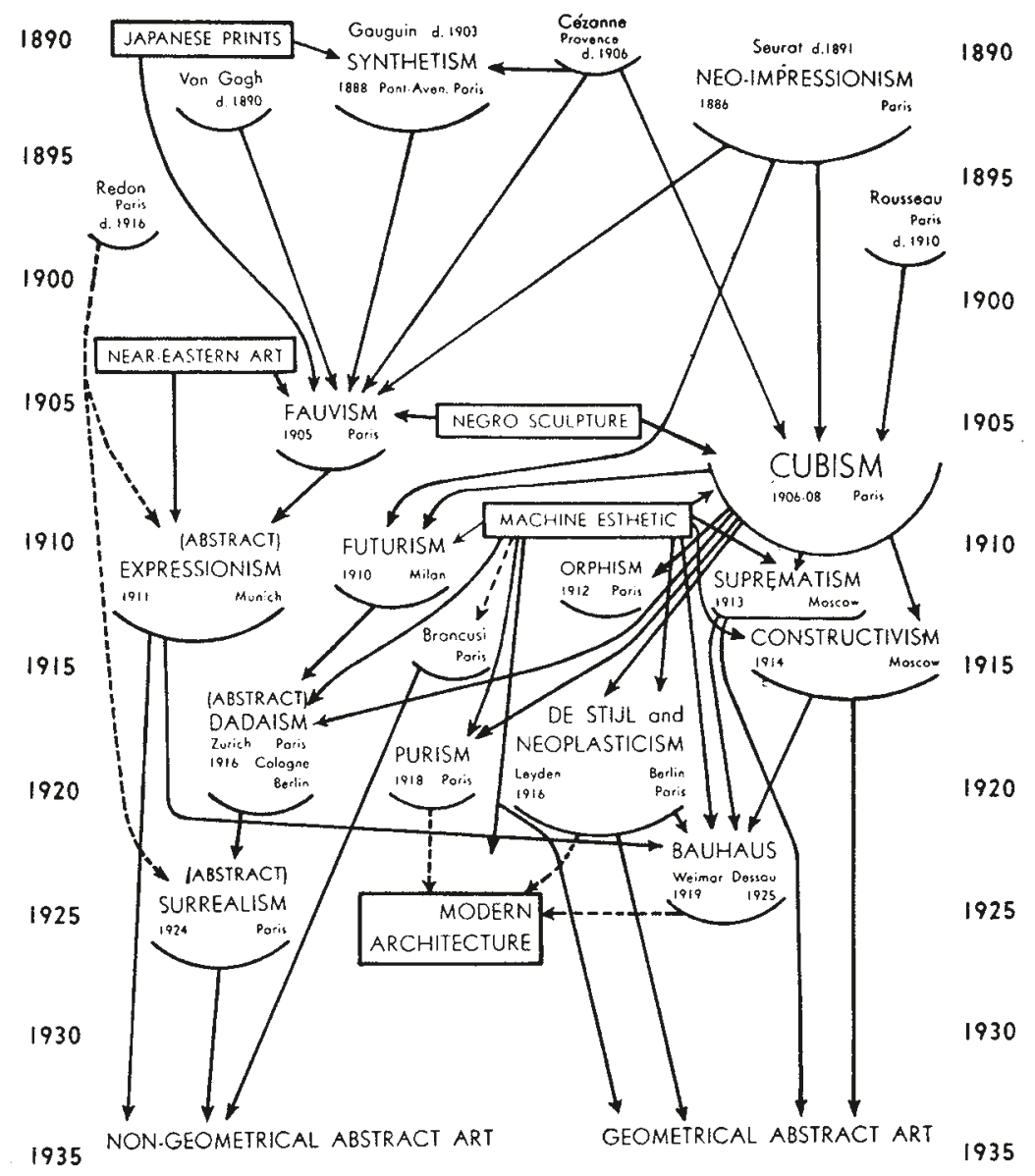

\section{CUBISM AND ABSTRACT ART}

Alfred H. Barr: Diagramm zur Stilentwicklung von 1890 bis 1935, 1936 